\title{
Aplication of The SMARTER Method for the Selection of The Best Ambassador Brand
}

\author{
Nasib Marbun ${ }^{1}$, Muhammad Zarlis ${ }^{2 *}$, Rahmat Widiä:Sembiring ${ }^{3}$ \\ ${ }^{1}$ Department of Computer Science, Universitas Sumatera Utara \\ ${ }^{2}$ Department of Computer Science, Universitas Sumatera Utara \\ ${ }^{3}$ Department of Computer Science, Politeknik Negeri Medan \\ ${ }^{1}$ marbunnasib93@gmail.com, ${ }^{2}{ }^{*}$ m.zarlis@usu.ac.id, ${ }^{3}$ rahmatws@polmed.ac.id
}

\begin{abstract}
This study aims to provide convenience for the company's management to solve problems in the process of selecting the best Brand Ambassador in promoting cosmetic tools. The problem identified in this study is the large number of prospective Brand Ambassadors, causing the management to find it difficult to determine the best Brand Ambassador. In this study, the decision support method applied is SMARTER which can determine the ranking results in the final decision using the level of importance of each parameter. The results of this study conclude that the selection of the best Brand Ambassador who is ranked first is A5 (Lucky) with a value of 0.653108466.
\end{abstract}

Keywords: Decision Support System, Brand Ambassador, Smarter

\section{Introduction}

Brand Ambassador is a term used for someone who is employed in a company to promote a product or service brand to the public [1]. Brand Ambassadors who are employed in a company must have a positive image that is able to attract people's attention to buy products or use the services offered [2].

The selection of the best Brand Ambassador for a cosmetic company is carried out through an assessment stage against the required criteria. In the study, it was found that the problem was that the large number of prospective Brand Ambassadors caused the management to find it difficult to determine the best Brand Ambassador. Solving the problems identified in the selection of the best Brand Ambassador experienced by the management of cosmetic companies in this study, the application of SMARTER was carried out. The SMART method is a development of SMART conducted by Edwards and Baron in 1994 [3][4]. The decision-making technique in the SMARTER Method is based on the Rank-Order Centroid (ROC) weighting formula [5][6].

\section{Research Methodology}

\subsection{Research Stages}

The stages of research that the author did are as follows:

a) Interview

The first stage, the author conducts interviews with takers in the company to obtain alternative data and the criteria used to determine the best Brand Ambassador.

b) Literature Review

The second stage, the author looks for references that are relevant to the research topic carried out to support each scientific statement used in this article.

c) Implementation Method

The third stage, the author implements the SMARTER method in the process of selecting the best Brand Ambassador.

d) Making Conclusions 


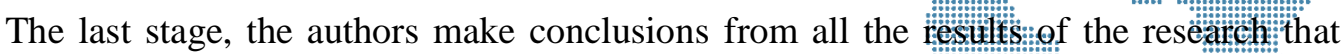
has been done.

\subsection{Simple Multiple Attribute Rating Technique Exploiting Ranks (SMARTER)}

SMARTER (Simple Multi-Attribute Rating Technique Exploiting Ranks) has a multicriteria decision-making technique that assumes that a number of criteria have different importance weight values from other criteria [7][8]. Determining the weight value in the SMARTER method is carried out based on a range from 0 to 1 using Rank-Order Centroid (ROC).

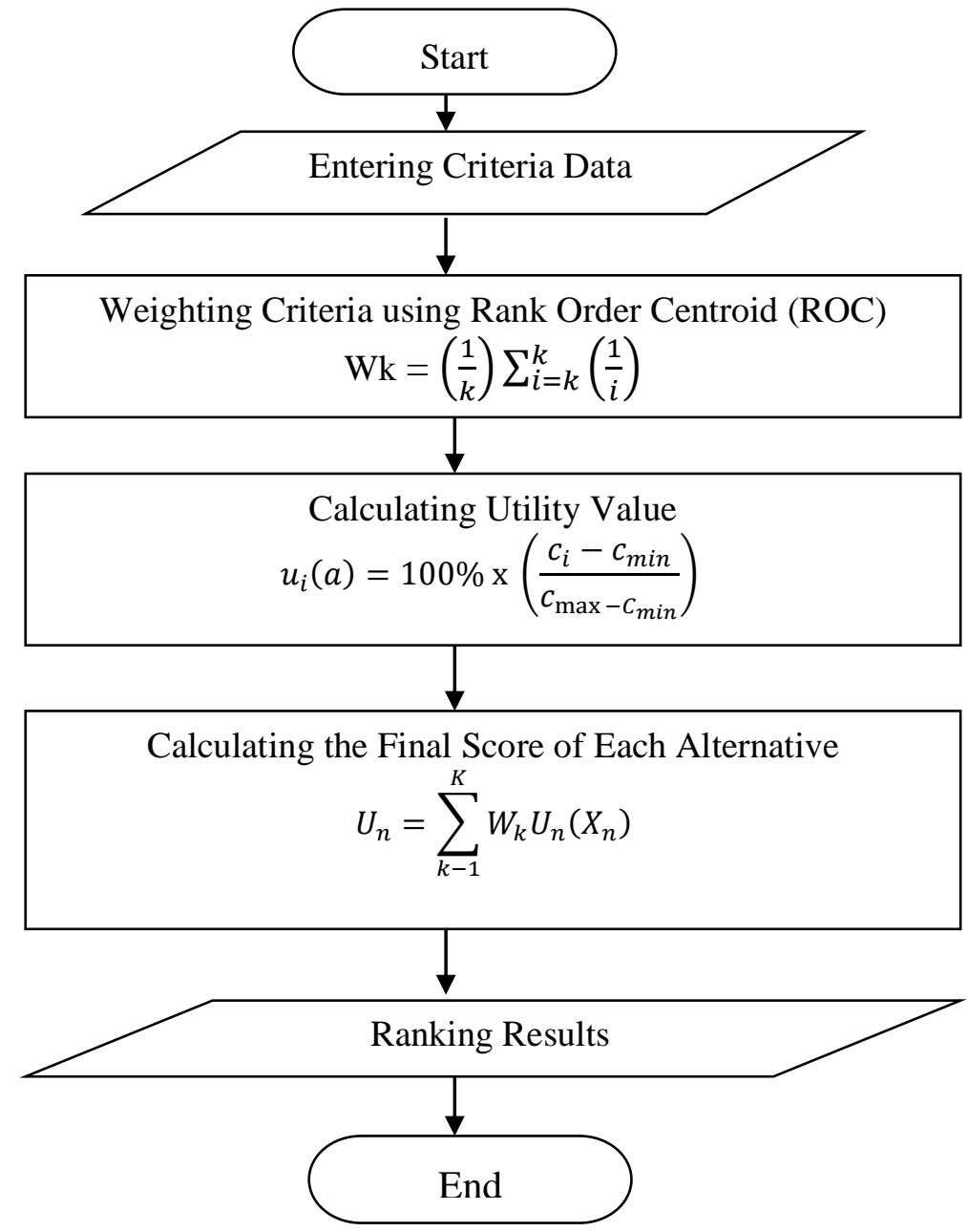

Figure 1. SMARTER Flowchart

\section{Result and Discussion}

Alternative data that will be used to select the best Brand Ambassador in this study can be seen in the following table :

Table 1. Alternative

\begin{tabular}{|c|c|}
\hline Code & Alternative \\
\hline A1 & Rian \\
\hline A2 & Despin \\
\hline A3 & Citra \\
\hline A4 & Yaskra \\
\hline A5 & Lucky \\
\hline
\end{tabular}

The next stage is implementing the SMARTER method for selecting the best Brand Ambassador using the following steps: 


\section{Csinta}

International Joüunal: of Information System: \& Téchiniology Akreditasi No. 36/E/KPT12019†Vol. 5, No. 2, (2021) "Pp:99403

1) Entering Criteria Data

The criteria data used for the selection of the best Brand Ambassadorstin this study can be seen in the following table :

Table 2. Criteria

Table 2. Criteria
\begin{tabular}{|c|c|}
\hline Code & Criteria \\
\hline C 1 & Attractiveness \\
\hline C 2 & Visibility \\
\hline C 3 & Credibility \\
\hline C 4 & Tech Adequate \\
\hline
\end{tabular}

2) Weighting Criteria using Rank Order Centroid (ROC) At this stage, the criteria are weighted using Rank Order Centroid (ROC) to determine the importance of the criteria used.

Table 3. Bobot Rank Order Centroid (ROC)

\begin{tabular}{|c|c|c|c|}
\hline \multirow{2}{*}{ Code } & \multirow{2}{*}{ Criteria } & \multicolumn{2}{|c|}{ Criteria Weight } \\
\cline { 3 - 4 } & & The Most Important & Least Important \\
\hline A1 & Professional Speaker & 60 & 50 \\
\hline A2 & Attractiveness & 50 & 40 \\
\hline A3 & Visibility & 40 & 30 \\
\hline A4 & Credibility & 30 & 20 \\
\hline \multicolumn{2}{|c|}{ Total Value } & 180 & 140 \\
\hline
\end{tabular}

Based on the most important and least important values in the Rank Order Centroid (ROC) table above, the average value of the weight value of each criterion is determined.

Table 4. Average Weight

\begin{tabular}{|c|l|l|c|}
\hline \multirow{2}{*}{ Criteria } & \multicolumn{3}{|c|}{ Criteria Weight } \\
\cline { 2 - 4 } & The Most Important & Least Important & Average \\
\hline C1 & $60 / 180=0.333333333$ & $50 / 140=0.357142857$ & 0.345238095 \\
\hline C2 & $50 / 180=0.277777778$ & $40 / 140=0.285714286$ & 0.281746032 \\
\hline C3 & $40 / 180=0.222222222$ & $30 / 140=0.214285714$ & 0.218253968 \\
\hline C4 & $30 / 180=0.166666667$ & $20 / 140=0.142857143$ & 0.154761905 \\
\hline
\end{tabular}

3) Calculating Utility Value

The next stage is to calculate the utility value of each paired matrix listed in the following table.

Table 5. Pairwise Matrix

\begin{tabular}{|c|c|c|c|c|}
\hline \multirow{2}{*}{ Alternative } & \multicolumn{4}{|c|}{ Criteria } \\
\cline { 2 - 5 } & C1 & C2 & C3 & C4 \\
\hline A1 & 0.5 & 0.6 & 0.7 & 0.6 \\
\hline A2 & 0.8 & 0.5 & 0.6 & 0.5 \\
\hline A3 & 0.4 & 0.8 & 0.5 & 0.6 \\
\hline A4 & 0.6 & 0.7 & 0.8 & 0.5 \\
\hline A5 & 0.7 & 0.6 & 0.7 & 0.8 \\
\hline Min & 0.4 & 0.5 & 0.5 & 0.5 \\
\hline Max & 0.8 & 0.8 & 0.8 & 0.8 \\
\hline
\end{tabular}

a. Professional Speaker

$$
\begin{aligned}
& \text { A1 }=100 \% \times\left(\frac{0.5-0.4}{0.8-0.4}\right)=0.25 \\
& \text { A2 }=100 \% \times\left(\frac{0.8-0.4}{0.8-0.4}\right)=1 \\
& \text { A3 }=100 \% \times\left(\frac{0.4-0.4}{0.8-0.4}\right)=0 \\
& \text { A4 }=100 \% \times\left(\frac{0.6-0.4}{0.8-0.4}\right)=0.5
\end{aligned}
$$

$$
\mathrm{A} 5=100 \% \times\left(\frac{0.7-0.4}{0.8-0.4}\right)=0.75
$$

b. Attractiveness

$$
\begin{aligned}
& \text { A1 }=100 \% \times\left(\frac{0.6-0.5}{0.8-0.5}\right)=0.333333 \\
& \text { A2 }=100 \% \times\left(\frac{0.5-0.5}{0.8-0.5}\right)=0 \\
& \text { A3 }=100 \% \times\left(\frac{0.8-0.5}{0.8-0.5}\right)=1
\end{aligned}
$$




\section{C) sînta}

$$
\begin{aligned}
& \text { A4 }=100 \% \times\left(\frac{0.7-0.5}{0.8-0.5}\right)=0.666667 \\
& \text { A } 5=100 \% \times\left(\frac{0.6-0.5}{0.8-0.5}\right)=0.333333
\end{aligned}
$$

c. Visibility

$$
\begin{aligned}
& \text { A1 }=100 \% \times\left(\frac{0.7-0.5}{0.8-0.5}\right)=0.6666667 \\
& \text { A2 }=100 \% \times\left(\frac{0.6-0.5}{0.8-0.5}\right)=0.3333333 \\
& \text { A3 }=100 \% \times\left(\frac{0.5-0.5}{0.8-0.5}\right)=0 \\
& \text { A4 }=100 \% \times\left(\frac{0.8-0.5}{0.8-0.5}\right)=1
\end{aligned}
$$

Table 6. Utility Calculation Results

\begin{tabular}{|c|c|c|c|c|}
\hline \multirow{2}{*}{ Alternative } & \multicolumn{4}{|c|}{ Criteria } \\
\cline { 2 - 5 } & C1 & C2 & C3 & C4 \\
\hline A1 & 0.25 & 0.333333 & 0.6666667 & 0.3333333 \\
\hline A2 & 1 & 0 & 0.3333333 & 0 \\
\hline A3 & 0 & 1 & 0 & 0.3333333 \\
\hline A4 & 0.5 & 0.666667 & 1 & 0 \\
\hline A5 & 0.75 & 0.333333 & 0.6666667 & 1 \\
\hline
\end{tabular}

4) Calculating the Final Score of Each Alternative

The next stage is to calculate the final value of each alternative based on the results of the calculation of the utility value.

a. Professional Speaker

$$
\begin{aligned}
& \text { A1 }=0.345238095 \times 0.25=0.086309524 \\
& \text { A2 }=0.345238095 \times 1=0.345238095 \\
& A 3=0.345238095 \times 0=0 \\
& A 4=0.345238095 \times 0.5=0.172619048 \\
& \text { A5 }=0.345238095 \times 0.75=0.258928571
\end{aligned}
$$

b. Attractiveness

$$
\begin{array}{ll}
A 1=0.281746032 & 0.333333=0.093915344 \\
A 2=0.281746032 & 0=0 \\
A 3=0.281746032 & 1=0.281746032 \\
A 4=0.281746032 & 0.666667=0.187830688 \\
\text { A5 }=0.281746032 & 0.333333=0.093915344
\end{array}
$$

c. Visibility

$$
\begin{array}{ll}
\text { A1 }=0.218253968 & 0.6666667=0.145502645 \\
\text { A2 }=0.218253968 & 0.3333333=0.072751323 \\
\text { A3 }=0.218253968 & 0=0 \\
\text { A4 }=0.218253968 & 1=0.218253968 \\
\text { A5 }=0.218253968 & 0.6666667=0.145502645
\end{array}
$$

d. Credibility

$$
\begin{array}{ll}
A 1=0.154761905 & 0.3333333=0.051587302 \\
A 2=0.154761905 & 0=0 \\
A 3=0.154761905 & 0.3333333=0.051587302 \\
A 4=0.154761905 & 0=0 \\
A 5=0.154761905 & 1=0.154761905
\end{array}
$$

Table 7. Calculating the Final Score of Each Alternative

\begin{tabular}{|c|c|c|c|c|c|}
\hline \multirow{2}{*}{ Alternative } & \multicolumn{4}{|c|}{ Criteria } & \multirow{2}{*}{ Final Score } \\
\cline { 2 - 5 } & C1 & C2 & C3 & C4 & \\
\hline A1 & 0.086309524 & 0.093915344 & 0.145502645 & 0.051587302 & 0.377314815 \\
\hline A2 & 0.345238095 & 0 & 0.072751323 & 0 & 0.417989418 \\
\hline A3 & 0 & 0.281746032 & 0 & 0.051587302 & 0.333333334 \\
\hline A4 & 0.172619048 & 0.187830688 & 0.218253968 & 0 & 0.578703704 \\
\hline
\end{tabular}




\begin{tabular}{|c|c|c|c|c|c|c|}
\hline \multirow{3}{*}{ Alternative } & \multirow{2}{*}{\multicolumn{4}{|c|}{ Criteria }} & \multirow{3}{*}{ Final Score: } & \multirow{3}{*}{ 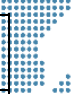 } \\
\hline & & & & & & \\
\hline & C1 & $\mathrm{C2}$ & C3 & G4: & & \\
\hline A5 & 0.258928571 & 0.093915344 & 0.145502645 & 0.154764905 & 0.653108466 & 耳": \\
\hline
\end{tabular}

5) Ranking Results

The ranking results obtained from the application of the SMARTER method for the selection of Brand Ambassadors can be seen in the table below.

Table 8. Ranking Results

\begin{tabular}{|c|c|c|}
\hline Alternative & Value & Rating \\
\hline A1 & 0.377314815 & 4 \\
\hline A2 & 0.417989418 & 3 \\
\hline A3 & 0.333333334 & 5 \\
\hline A4 & 0.578703704 & 2 \\
\hline A5 & $\mathbf{0 . 6 5 3 1 0 8 4 6 6}$ & $\mathbf{1}$ \\
\hline
\end{tabular}

\section{Conclusion}

In the results of this study it was concluded that the SMARTER method can provide a range of values between the alternative and the measurable so that it can produce a good rating, the alternative that gets the first rank is A5 (Lucky) with a value of 0.653108466 or the highest value among other alternatives, the SMARTER method can be used as the best alternative to support decision making in selecting the best Brand Ambassador Brand.

\section{References}

[1] G. Geraldine and D. A. Candraningrum, "Pengaruh Kredibilitas Brand Ambassador Non-Celebrity Analisa Widyaningrum terhadap Keputusan Pembelian Hijabenka," Prologia, vol. 4, no. 1, p. 25, 2020, doi: 10.24912/pr.v4i1.6422.

[2] E. M. Fitrianto, . N., and . I., "Brand Ambassador Performance and the Effect to Consumer Decision Using VisCAP Model on Online Marketplace in Indonesia," Sriwij. Int. J. Dyn. Econ. Bus., vol. 4, no. 1, p. 21, 2020, doi: 10.29259/sijdeb.v4i1.21-30.

[3] I. Santosa, D. A. Wijaya, Mulaab, A. Rachmad, and T. Novianti, "Development of Poor Family Selection System Using Simple Multi Attribute Rating Technique Exploiting Rank Method," J. Phys. Conf. Ser., vol. 1569, no. 2, 2020, doi: 10.1088/1742-6596/1569/2/022082.

[4] N. Silalahi, P. Studi, and T. Informatika, "Bulletin of Information Technology ( BIT ) Sistem Pendukung Keputusan Pemilihan Dosen Berprestasi Menggunakan Metode SMARTER Pada Universitas Budi Darma," vol. 1, no. 1, pp. 50-57, 2020.

[5] M. Kusmiyanti, Richa Dwi, Suliatun, "Analisis Sensitifitas Model SMART-AHP dengan SMARTER-ROC sebagai Pengambilan Keputusan Multi Kriteria," Semin. Nas. Teknol. Informasi, Komun. dan Ind. 9, pp. 18-19, 2017.

[6] S. S. Helma et al., "Penerapan Metode SMARTER untuk Penentuan Hasil Open Recruitment Anggota Puzzle Research Data Technology ( Predatech )," no. November, pp. 277-285, 2019.

[7] N. M. Sitinjak and M. Zarlis, "Analisis Decision Support System Perbandingan Metode Smarter dan Saw Dalam Menentukan Pemilihan Staff Pegawai Terbaik Laboratorium Komputer," vol. 5, no. April, pp. 495-503, 2021, doi: 10.30865/mib.v5i2.2926.

[8] R. Rasim, Rahman, Dewi, "Decision Support Systems for Performance and Evaluation of Teachers in General-English Course by Using the SMARTER and TOPSIS Methods," IOP Conf. Ser. Mater. Sci. Eng., vol. 755, no. 1, 2017, doi: 10.1088/1742-6596/755/1/011001. 